\title{
The knowledge of hospital patients about vascular diseases and their risk factors
}

\author{
SE Gariballa, SM Peet, MD Fotherby, SG Parker, CM Castleden
}

\begin{abstract}
Summary
The knowledge of 28 stroke patients on the nature, consequences, treatment and risk factors of stroke and ischaemic heart disease was examined using a questionnaire and compared with that of 26 patients with ischaemic heart disease and 41 controls without evidence of vascular disease. Information was also collected on the patients' willingness to change their life-style, the information and advice they had received and their desire for more information. It was found that about half of the elderly stroke and heart disease patients had a reasonable knowledge of the condition and its related risk factors. Only eight (14\%) patients remembered receiving information and advice in relation to their condition during their hospital stay compared with one $(2 \%)$ control. There was a significant difference between the number of stroke and heart disease patients who wanted to know more about their condition compared with the control group (32 vs 14; $p=0.03$ ). A quarter of the patients and half of the controls knew that fruit and vegetables were good for you and excessive fat and alcohol were less inducive to good health. Most patients with a risk factor were willing to exercise more, stop smoking, cut down on their drinking, or lose weight. These results suggest that elderly hospital patients have a reasonable basic knowledge about vascular diseases, but that a significant number want to know more and would be willing to change their life-style.
\end{abstract}

Department of Geriatrics, The Hayward Building, Selly Oak Hospital, Raddlebarn Road, Birmingham B29 6JD, UK

SE Gariballa

University Division of Medicine for the Elderly, Leicester General Hospital, Gwendolen Road, Leicester LE5 4PW, UK

SM Peet

MD Fotherby

SG Parker

CM Castleden

Accepted 27 February 1996
Keywords: vascular disease, patient knowledge

It has been known for some time that patients are poorly informed about their illnesses whilst in hospital, and this lack of information might have far-reaching implications in terms of specific disease management strategies. ${ }^{1-3}$ Stroke and ischaemic heart disease patients are not exceptional in this regard. ${ }^{4-5}$ Stroke, for example, imposes a heavy work load on hospital and community care services, and life after stroke can be a miserable affair for the majority of sufferers who are left with residual disability. ${ }^{6}$ Measures which can lower the incidence or improve the clinical outcome of stroke would therefore be welcomed. Many of these measures require client co-operation which will be more likely if there were an understanding of the underlying principles of risk-reducing methods. Patients who understand and comply with medical advice can substantially alter their risk of disease. Compliance with hypotensive therapy, ${ }^{7}$ regular exercise post-myocardial infarction, ${ }^{8}$ and tight glycaemic control in diabetes mellitus ${ }^{9}$ are examples.

There are no good randomised trials of secondary risk factor modification in stroke patients, but such factors are well known and inference could be drawn from primary prevention studies which include control of blood pressure, anticoagulation of patients with atrial fibrillation, discouragement of cigarette smoking, and reduction of excessive alcohol consumption. ${ }^{10}$ Health professionals can play an important role in primary and secondary prevention of stroke by identifying patients at risk, supplying information, prescribing appropriate drugs, providing monitoring and giving support. We therefore decided to evaluate whether stroke patients were adequately informed about the nature, consequences, treatment and risk factors of stroke, and to compare their knowledge with that of patients suffering from ischaemic heart disease and a control group without evidence of vascular disease. We also asked whether they would be willing to change their life-style in order to reduce any risk factors.

\section{Patients and methods}

During a period of six months 114 patients on two general medical and two rehabilitation wards consented to be interviewed. They were non-randomly selected consecutive admissions to our unit, of whom 38 were stroke patients according to the standard definition of the World Health Organisation, ${ }^{11} 31$ were admitted to hospital because of ischaemic heart disease (five had a stroke and ischaemic heart disease); the remaining 45 had a wide variety of diseases including chest and urinary tract infections, rheumatoid and osteoarthritis, Parkinson's disease and gastrointestinal disorders but no clinically detectable vascular disease on history, examination, electrocardiogram and chest X-ray. They were comparable to the stroke and ischaemic heart disease group in terms of age, time of hospital admission, cognitive function and duration of hospital stay. The interviews were conducted, on average, two weeks after admission. Patients who were unable to co-operate because of impaired cognition or speech were excluded. 
Three patients refused to be interviewed. This study had the statistical power to detect a difference of $30 \%$ in response to questionnaire items between groups at the $5 \%$ level with a $20 \%$ probability of type 2 error.

\section{QUESTIONNAIRE DEVELOPMENT}

A questionnaire was designed to cover areas of general knowledge of predisposing factors, the nature, consequences, implications and treatment of stroke and heart attack. Information was also collected on the patients' willingness to change their life-style in order to modify their risk factors, the information and advice they had received, and their desire for more information.

The questionnaire was piloted on 20 patients on two different occasions. Changes were made according to the responses obtained from the patients, interviewers and colleagues.

The interviews were conducted by two experienced healthcare research interviewers. Before the beginning of the actual study and for assessment of inter-rater agreement 10 patients were interviewed initially by each interviewer and the inter-rater agreement was $\mathrm{K}=0.82$ using the kappa measure of agreement from a table of frequencies.

\section{ANALYSIS OF RESULTS}

Each patient's response was coded for each question on a scale from 1 for a correct answer, 2 for an incorrect answer to 3 for 'don't know' and organised into categories. For each section the correct scores were added, a mean obtained and the results quoted as numbers of patients with a correct score. Questionnaire items to which the responses were descriptive were excluded from the analysis. Differences between the stroke, ischaemic heart disease and control groups were explored using the Mann Whitney U test, $\chi^{2}$, and Kruskall Wallis, where appropriate. Yates' correction was used when the sample size was small or Fisher's exact test when one cell had an expected frequency of less than 5 .

\section{Results}

A total of 19 of 114 patients interviewed were excluded from the analysis because of incomplete data (10 with stroke, five with ischaemic heart disease and four controls). Of the remaining 95 patients, 28 had stroke, 26 ischaemic heart disease and 41 had a wide variety of diseases other than stroke, ischaemic heart disease or peripheral vascular disease. The patients' age and sex in each group were

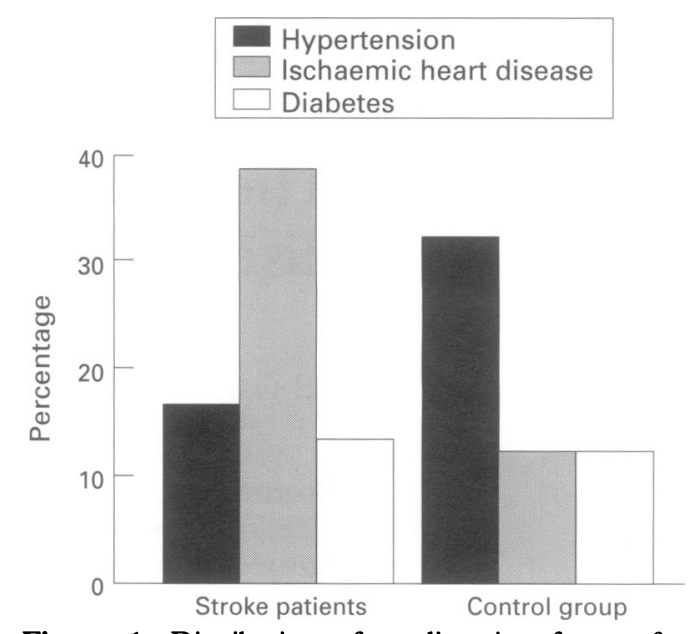

Figure 1 Distribution of predisposing factors for $\vec{\circ}$ vascular disease in stroke patients and controls. There was a statistically significant difference between groups for ischaemic heart disease $(p<0.05)$

similar (mean age $75 \pm 8$ years and $54 \%$ were female).

Patients in the stroke and control groups were compared with respect to the distribution of of predisposing factors for vascular disease. More of the stroke patients than controls had concurrent ischaemic heart disease $(p<0.05)$ but, surprisingly, more controls than stroke patients suffered from hypertension (figure 1).

GENERAL KNOWLEDGE OF VASCULAR DISEASE The questions in this section were mainly about the causes, consequences and treatment of stroke and ischaemic heart disease. More than $50 \%$ of the questions were answered correctly by $24(44 \%)$ ischaemic heart disease and stroke patients compared with $15(37 \%)$ of the control group $(p=0.6$; see table and figure 2 ). There was no significant difference between stroke $(46 \%)$ and ischaemic heart disease $(42 \%)$ patients $(p=0.17)$

\section{KNOWLEDGE OF RISK FACTORS}

Questions also examined the knowledge of risk factors which predispose to stroke and ischaemic heart disease, such as being overweight, having high blood pressure, smoking, drinking 우 excessive alcohol and taking little exercise. $D$ Lack of sleep and having the flu or a cold were included as dummy questions. Over half (28) N of the stroke and ischaemic heart disease patients correctly identified more than two of $\mathcal{N}$ five risk factors compared to $23(56 \%)$ controls $(p=0.84)$. There was no difference between stroke and ischaemic heart disease patients in this regard (see table and figure 2).

Table Number of stroke, ischaemic heart disease patients and controls with correct (appropriate) response

\begin{tabular}{llll}
\hline & $\begin{array}{l}\text { Ischaemic heart disease } \\
(n=26)\end{array}$ & $\begin{array}{l}\text { Stroke } \\
(n=28)\end{array}$ & $\begin{array}{c}\text { Control } \\
(n=41)\end{array}$ \\
\hline General knowledge & 11 & 13 & 15 \\
Knowledge of risk factors & 14 & 14 & 23 \\
Those given information & 5 & 3 & 1 \\
Those wanting to know more & 17 & 15 & 14 \\
\hline
\end{tabular}




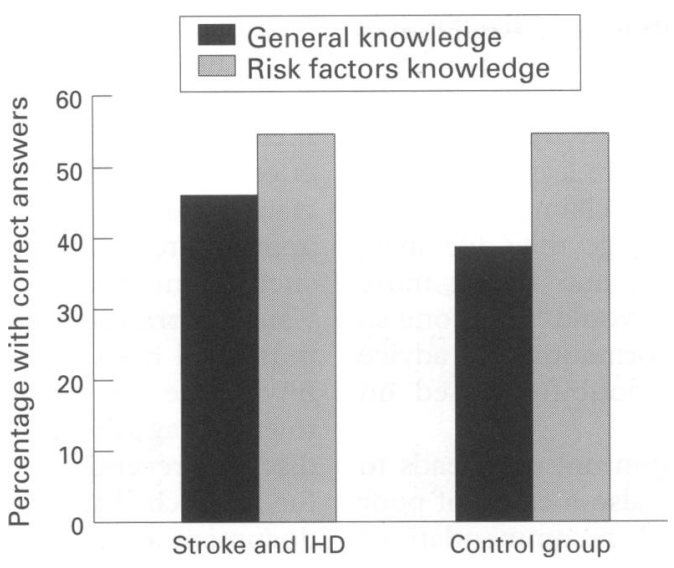

Figure 2 General knowledge about vascular disease and its risk factors in stroke and ischaemic heart disease (IHD) patients compared to controls

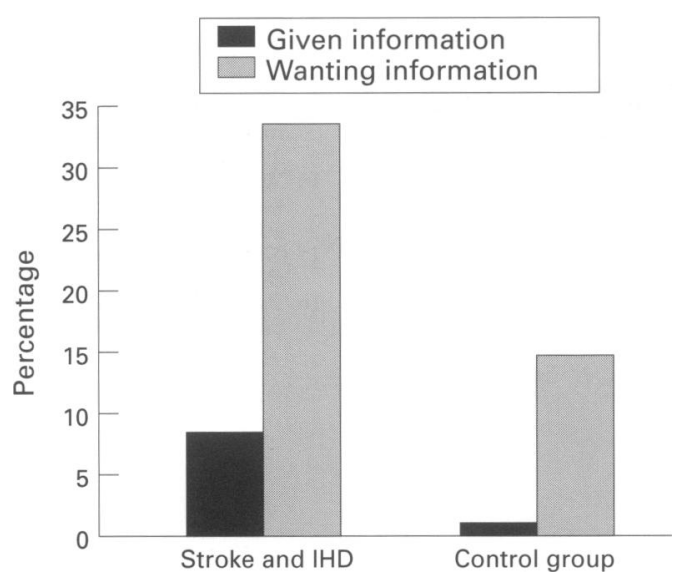

Figure 3 Information and advice on vascular disease given to stroke and ischaemic heart disease (IHD) patients compared to controls. The differences between the groups were statistically significant (see text)

\section{INFORMATION AND ADVICE}

Eight (14\%) stroke or ischaemic heart disease patients remembered receiving information and advice in relation to their condition during their hospital stay compared to one $(2 \%)$ control $(p=0.07)$; there was no significant difference between stroke (11\%) and ischaemic heart disease $(19 \%)$ patients. Four $(10 \%)$ controls could not remember whether or not they had received information. Significantly more stroke and ischaemic heart disease patients wanted to know more about their condition and how to prevent a recurrence than patients in the control group (32 vs 14, $\mathrm{p}=0.03$; figure 3 ).

DIET, BODY WEIGHT, SMOKING AND EXERCISE When asked about the effects of various dietary components on vascular status, $15(28 \%)$ stroke and ischaemic heart disease patients gave an appropriate response compared to 20 $(49 \%)$ controls $(p=0.2)$. Ten $(71 \%)$ of those who admitted to smoking said they would be prepared to stop smoking provided they had access to some help. Irrespective of their current body weight, $26(27 \%)$ were prepared to lose weight if advised to do so by a doctor.
Thirty-eight (40\%) patients thought they took enough exercise but 55 (58\%) said they would exercise more if doing so would make them healthier or reduce their chances of having a stroke or ischaemic heart disease. No one admitted to drinking more than 16 units of alcohol per week but $26(27 \%)$ were prepared to cut down on their drinking if advised.

\section{Discussion}

A possible explanation for the decrease in stroke mortality rates in recent years is a change in the level of risk factors. ${ }^{10}$ However, there is still room for improvement, and further risk-factor modification could decrease the number of strokes in the community and the associated disability and handicap. ${ }^{12}$ The traditional approach to prevention of stroke and ischaemic heart disease is to identify people at risk at the individual and population level and modify their risk factors by treatment and education.

About half of the elderly stroke patients had a good knowledge of their condition and its related risk factors. This would suggest a reasonable degree of basic knowledge in the population at large. However, very few remembered receiving information or advice in relation to their condition during their hospital stay, which reinforces an earlier publication from this centre suggesting that risk factors had not been addressed by hospital staff. ${ }^{13}$ It was surprising in view of the public information on heart diseases and recent improvements in the organisation of cardiac services that the knowledge and information given to patients with ischaemic heart disease was no better than that given to stroke patients. Patients without ischaemic heart disease or stroke confirmed that the general knowledge about these conditions and their risk factors in the population at large was reasonable, as they had the same levels as the patients with those conditions. These patients had not received any specific information about stroke and heart conditions.

Although there are methodological differences between the study of Wellwood $e t$ al and the present study, Wellwood also found that many stroke patients and their carers want more information and discussion about their illness and treatment during their hospital stay. ${ }^{14}$ The Gallup poll commissioned by the Stroke Association also found that a large proportion of the UK adult population were uncertain of what a stroke was or how to prevent it. ${ }^{14}$ It is indeed significant that many patients with stroke and ischaemic heart disease in our study could not correctly identify the risk factors for either condition, even after they had been in hospital. Such a lack of information and communication are contrary to the standards set out by the King's Fund Consensus. ${ }^{15}$ Clearly there are problems associated with recall and there were also problems associated with low numbers. Nevertheless, the present results suggest that more education is required if secondary prevention of disease is to be a realistic aim.

Just how far present educational pro- 
grammes work is doubtful, as only about a quarter of patients who had had a stroke or ischaemic heart disease and half the controls knew that fruit and vegetables were good for you while fat and alcohol were less inducive to good health. Many patients claimed that they would be prepared to change their life-style, such as stopping smoking and taking more exercise, but whether they would have done so simply on being given information and advice about their condition is doubtful, based on previous studies. ${ }^{16}$

Failure in communication not only leads to dissatisfied patients but is also a cause of poor compliance with doctors' recommendations

1 Reynolds $M$. No news is bad news: patients' views about

2 Dunkelman H. Patients' knowledge of their condition and 2 Dunkelman H. Patients' knowledge of their condition and treatment: how it might be improved. $B M F$ 1979; $2: 311-4$. 281: $845-6$.

4 Hanger HC, Mulley GP. Questions people ask about stroke. Stroke 1993; 24: 536-8.

5 Lomer M, McLennan DL. Informing hospital patients and their relatives about stroke. Clin Rehab 1987; 1 : 33-7.

6 Holbrook M. Stroke: social and emotional outcome. $f R$ Coll Physic Lond 1982; 16: 100-4.

7 Balazovjech I, Hnilica P Jr. Compliance with antihypertensive treatment in consultation room for hypertensive patients. F Hum Hypertens 1993; 7: 581-3.

8 Fletcher GF. Exercise in the prevention of stroke. Health Rep 1994; 6: 106-9.

9 The 1994; 6: $106-9$. Group. The effect of intensive treatment of diabetes on the Group. The effect of intensive treatment of diabetes on the development and progression of long-term complications in 329: $977-86$. for treatment and changes in life-style. This study has shown that hospital patients are not well informed and also reveals that the existing methods of information delivery are not effective. Health education through a physician-nurse team approach, supported by appropriate methods of information delivery such as increasing available literature, audiovisual information packages, discussion on an individual basis, group meetings and community stroke care officers, are some of the ways to encourage the changes that support vascular disease prevention. This is an important area for research if the aims of Health of the nation document are to be realised.

10 Bonita R. Epidemiology of stroke. Lancet 1992; 339: 342 -

11 World Health Organisation. Cerebrovascular disease prevention, treatment and rehabilitation. Technical report series no 469. Geneva: WHO, 1971.

12 Dunbabin D, Sandercock P. Preventing stroke by the modification of risk factors. Stroke 1990; 21 (suppl iv): 36-9.

13 Gariballa SE, Robinson TG, Parker SG, Castleden CM. A prospective study of primary and secondary risk factor management in stroke patients. $\mathcal{f} R$ Coll Physic Lond 1995 29: $485-7$.

14 Wellwood I, Dennis MS, Warlow CP. Perception and knowledge of stroke among surviving patients with stroke and their carers. Age Ageing 1994; 23: $293-8$.

15 King's Fund Consensus Statement. Treatment of stroke. KMf 1988; 297: 126-8.

16 Pain HSB, McLellan DL. The use of individualized Pain HSB, McLellan DL. The use of individuter
booklets after stroke. Clin Rehab 1990; 4: 265-72. 